\title{
Compressive Object Tracking using Entangled Photons
}

\author{
Omar S. Magaña-Loaiza, ${ }^{1, \text { a) }}$ Gregory A. Howland, ${ }^{2}$ Mehul Malik, ${ }^{1}$ John C. Howell, ${ }^{2}$ and \\ Robert W. Boyd ${ }^{1, b)}$ \\ 1) The Institute of Optics, University of Rochester, Rochester, NY 14627, \\ $U S A$ \\ ${ }^{2)}$ Department of Physics and Astronomy, University of Rochester, Rochester, \\ $N Y 14627, U S A$
}

(Dated: 31 October 2018)

\begin{abstract}
We present a compressive sensing protocol that tracks a moving object by removing static components from a scene. The implementation is carried out on a ghost imaging scheme to minimize both the number of photons and the number of measurements required to form a quantum image of the tracked object. This procedure tracks an object at low light levels with fewer than $3 \%$ of the measurements required for a raster scan, permitting us to more effectively use the information content in each photon.
\end{abstract}

a) omar.maganaloaiza@rochester.edu

b) Also at Department of Physics, University of Ottawa, Ottawa, Ontario K1N 6N5, Canada. 
Compressive sensing (CS) has recently been of great utility in quantum optical and lowlight level applications, for instance, single-photon level imaging, entanglement characterization and ghost imaging ${ }^{1-5}$. CS provides a resource-efficient alternative to single-photon arrayed detectors, permitting us to reduce operational problems involved in systems employing raster scanning ${ }^{6}$.

CS applies optimization to recover a signal from incomplete or noisy observations of the original signal through random projections ${ }^{7}$. These ideas applied to the field of imaging allow one to retrieve high resolution images from a small number of measurements ${ }^{8}$. Recently, the quantum optics community has employed CS for quantum state tomography, ${ }^{9,10}$ to demonstrate nonclassical correlations ${ }^{4}$ and to form compressed ghost images ${ }^{6}$.

Ghost imaging is a technique which employs the correlations between two light fields to reproduce an image. For example, entangled photons exhibit strong correlations in many properties such as time-energy and position-momentum ${ }^{11}$. One photon of an entangled pair illuminates an object and is collected by a bucket detector, which does not provide spatial information. Its entangled partner photon is then incident on a spatially resolving detector gated by the first photon's bucket detector. Remarkably, an image of the object appears on the spatially resolving detector, even though its photon never directly interacted with the object $^{12}$.

Compressive ghost imaging ${ }^{5}$ allows one to replace the spatially resolving detector with a bucket detector. This procedure reduces both acquisition times for systems based on raster scanning and the required number of measurements for retrieving images ${ }^{6}$. These improvements have motivated an ongoing effort to implement technologies based on ghost imaging such as image encryption ${ }^{13}$, quantum sensors ${ }^{14}$, object identification ${ }^{15}$ and most recently ghost imaging ladar ${ }^{16}$.

In spite of the advantages that technologies based on ghost imaging offer, they can be hard to implement in practice. Most current quantum optical technologies work at the single photon level, and are unfortunately vulnerable to noise and are inefficient, requiring many photons and many measurements ${ }^{17}$. To reduce these limitations, we apply an efficient form of compressive sensing. This allows us to overcome the main problems which undermine the practical application of many attractive correlated optical technologies. To demonstrate these improvements, we implement a ghost object tracking scheme that significantly outperforms traditional techniques. This opens the possibility of using correlated light in realistic 
applications for sparsity-based remote-sensing.

We present a proof-of-principle experiment based on a quantum ghost imaging scheme that allows us to identify changes in a scene using a small number of photons and many fewer realizations than those established by the Nyquist-Shannon criterion. ${ }^{18}$ Object tracking and retrieval is performed significantly faster in comparison to previous protocols ${ }^{5,6,12,19-21}$. This scheme uses compressive sampling to exploit the sparsity of the relative changes of a scene with a moving object. With this approach we can identify the moving object and reveal its trajectory. Our strategy involves removing static components of a scene and reduces the environmental noise present during the measurement process. This leads to the reduction of the number of measurements that we take and the number of photons required to form an image, both important issues in proposals for object tracking and identification ${ }^{15,19}$. The reduction of noise and removal of static components of a scene is carried out by subtracting two observation vectors, corresponding to two realizations of a scene. We call this technique ghost background subtraction. Our results demonstrate that this technique is adequate for object tracking at low light levels.

Consider the ghost imaging scheme depicted in Fig. 1. A laser pumps a nonlinear crystal oriented for type-I spontaneous parametric down-conversion (SPDC). The approximated output state is given by first order perturbation theory, which leads us to the following two-photon entangled state:

$$
|\Psi\rangle=\int d \vec{k}_{g} d \vec{k}_{o} f\left(\vec{k}_{g}+\vec{k}_{o}\right) \hat{a}_{g}^{\dagger}\left(\vec{k}_{g}\right) \hat{a}_{o}^{\dagger}\left(\vec{k}_{o}\right)|0\rangle
$$

We refer to the down-converted photons as the ghost and object photons denoted by the subindices $g$ and $o$, respectively. The two-photon probability amplitude, which is responsible for the transverse momentum correlations existing between the ghost and object photons, is represented by the non-factorizable function $f\left(\vec{k}_{g}+\vec{k}_{o}\right)$, where $k$ is the transverse wavevector of the ghost or object photon. The form of this function depends on the phase-matching conditions, but it is often approximated by a double gaussian function ${ }^{22}$. This two-photon entangled state is strongly anti-correlated in transverse momentum, such that if the transverse momentum of the object photon is measured, the transverse momentum of the ghost photon is found to have the same magnitude and opposite direction. These momentum anti-correlations allow us to perform ghost imaging.

In our experiment, we use digital micromirror devices (DMDs) to impress spatial infor- 


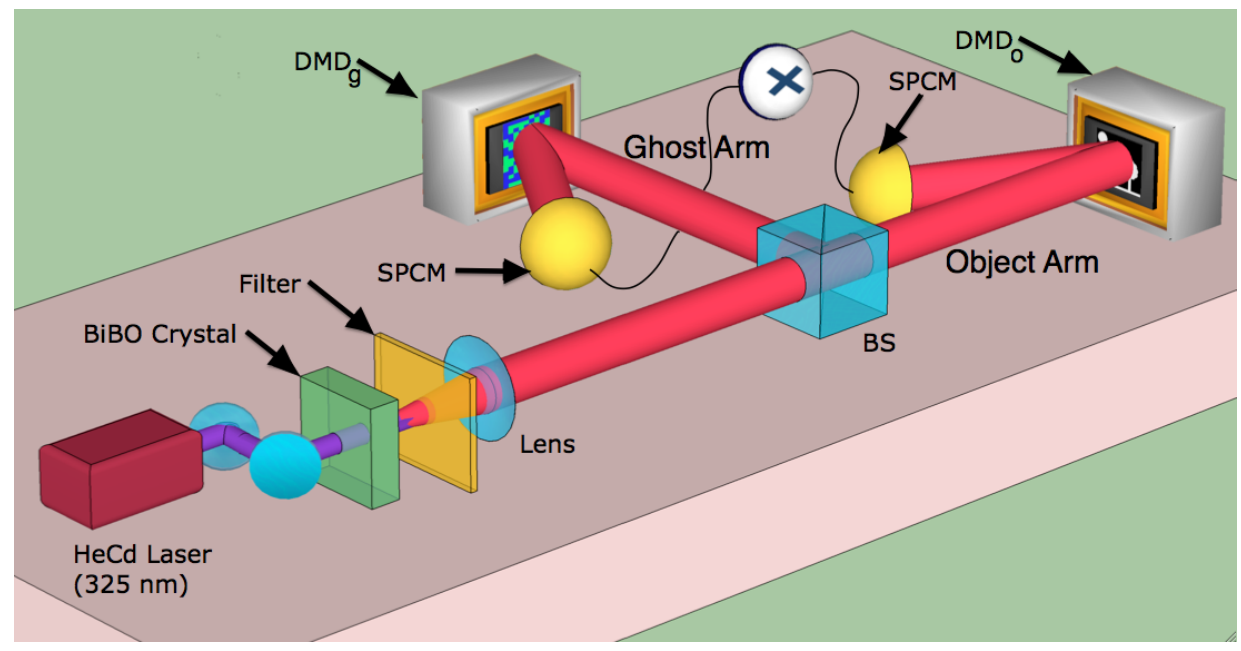

FIG. 1. Entangled photons at $650 \mathrm{~nm}$ are generated in a Bismuth Barium Borate (BiBO) crystal through type-I degenerate spontaneous parametric downconversion (SPDC). The far field of the BiBO crystal is imaged onto two digital micromirror devices (DMDs) with a lens and a beam splitter (BS). One DMD is used to display the object we want to track, while the other is used to display random binary patterns. Single-photon counting modules (SPCMs) are used for joint detection of the ghost and object photons.

mation onto the entangled photon pair. The DMDs work by controlling the retro-reflection of each individual pixel on the display. After each photon is reflected by a DMD, a singlephoton counting module (SPCM) counts the number of photons in it. The correlations between the two down-converted photons allows one to correlate the images displayed in the DMDs.

We jointly detect photons pairs reflected off a changing scene $O$ and a series of random matrices $A_{m}$. The subindex $m$ indicates the $m$-th realization. The coincidence counts between the two detectors are given by

$$
J_{m} \propto \int d \vec{\rho}_{\mathrm{DMD}}\left|A_{m}\left(\frac{\vec{\rho}_{\mathrm{DMD}}}{m_{r}}\right)\right|^{2}\left|O\left(\frac{-\vec{\rho}_{\mathrm{DMD}}}{m_{o}}\right)\right|^{2},
$$

where $A_{m}$ and $O$ are the reflectivity functions displayed on the $\mathrm{DMD}_{g}$ located in the ghost arm and on $\mathrm{DMD}_{o}$ in the object arm, respectively. Meanwhile $m_{r}$ and $m_{o}$ are their corresponding magnification factors. These are determined by the ratio of the distance between the nonlinear crystal to the lens and the distance from the lens to $\mathrm{DMD}_{g}$ or $\mathrm{DMD}_{o}$. 
In our experiment $m_{r}$ and $m_{o}$, are equal. $\vec{\rho}_{\mathrm{DMD}}$ represents the transverse coordinates of one of the DMDs.

Eq. 2 critically shows that the joint-detection rate is proportional to the spatial overlap between the images displayed on $\mathrm{DMD}_{o}$ and $\mathrm{DMD}_{g}$. This behavior can be interpreted as a nonlocal projection, which demonstrates the suitability for implementing compressive sensing techniques nonlocally with ghost imaging ${ }^{6}$.

Compressive sensing uses optimization to recover a sparse $n$-dimensional signal from a series of $m$ incoherent projective measurements, where the compression comes from the fact that $m<n$. Image reconstruction via compressive sensing consists of a series of linear projections $^{23}$. Each projection is the product of the image $O$ consisting of $n$ pixels, with a pseudorandom binary pattern $A_{m}$. Each pattern produces a single measurement, which constitutes an element of the observation vector $J$. After a series of $m$ measurements, a sparse approximation $\hat{O}$ of the original image $O$ can be retrieved by solving the optimization problem, known as total variation minimization ${ }^{24}$, given by Eq. 3 .

$$
\min _{\hat{O} \in C^{n}} \sum_{i}\left\|D_{i} \hat{O}\right\|_{1}+\frac{\mu}{2}\|A \hat{O}-J\|_{2}^{2} .
$$

$D_{i} \hat{O}$ is a discrete gradient of $\hat{O}$ at pixel $i, \mu$ is a weighting factor between the two terms, and $A$ is the total sensing matrix containing all the pseudorandom matrices $A_{m}$. Each matrix $A_{m}$ is represented into a $1 \mathrm{D}$ vector and constitutes a row of the total sensing matrix A. The algorithm known as Total Variation Minimization by Augmented Lagrangian and Alternating Direction (TVAL3) allows us to solve the aforementioned problem. The solution

of the optimization problem allows us to recover the image $\hat{O}$, which is the compressed version of the original image $O$, with a resolution given by the dimensions of the matrix $A_{m}$. The original image $\mathrm{O}$ is characterized by a sparsity number $k$, which means that the image can be represented in a certain sparse basis where $k$ of its coefficients are nonzero. The number of performed measurements $m$ is greater than the sparsity number $k$, but far fewer than the total number of pixels $n$ contained in the original image. The constraints imposed in the recovery algorithm minimize the noise introduced during the measurement process.

We are able to compressively track and identify a moving object in a scene by discarding static pixels. A scene with a moving object possesses static elements that do not provide 
information about the object's motion or trajectory. These redundancies can be discriminated from the moving object as follows. Let us consider the projection of two different frames onto the same pseudorandom pattern. Each projective measurement picks up little information about the components of a frame. If the two projective measurements produce the same correlation value, it would imply that the two frames are identical and we are retrieving meaningless information which can be ignored. The opposite case would reveal information about the changes in a scene.

This protocol is formalized as follows. Two different correlation vectors, $J^{j}$ and $J^{j-1}$, corresponding to two consecutive frames are subtracted, giving $\Delta J$. This introduces the following important modification to Eq. 3.

$$
\min _{\hat{O} \in C^{n}} \sum_{i}\left\|D_{i} \Delta \hat{O}\right\|_{1}+\frac{\mu}{2}\|A \Delta \hat{O}-\Delta J\|_{2}^{2} .
$$

The subtracted vector $\Delta J$ is sparser than both $J^{j}$ or $J^{j-1}$, thus requiring fewer measurements for its reconstruction. This corresponds to fewer realizations of $A_{m}$, and hence smaller sensing matrix $A$. Furthermore, subtracting the background in this manner mitigates the environmental noise present during the tracking process. The retrieved image $\Delta \hat{O}$ will provide information about the relative changes in the scene.

Our experimental setup is sketched in Fig. 1. A $325 \mathrm{~nm}$, continuous-wave HeCd laser pumps a type-I phase matched BiBO crystal to produce degenerate entangled photon pairs at $650 \mathrm{~nm}$. Two interference filters are placed after the nonlinear crystal. The first is a low pass filter that removes the pump and the second is a $650 / 12 \mathrm{~nm}$ narrowband filter that transmits the down-converted photons. A beam splitter probabilistically separates the two photons into ghost and object modes. An $88 \mathrm{~mm}$ focal length lens puts the far field of the crystal at the location of DMD. Two free space detectors receive the light reflected from the DMDs by means of two collection lenses with a $25 \mathrm{~mm}$ focal length. One DMD is used to display a scene with a moving object while the other is used to impress a series of random binary patterns. Coincidence counts are obtained within a 3 ns time window.

We apply this method to a scene with a flying object. The static components of the scene are a house, the moon and a tree. The object moves a certain distance in each iteration of the scene (insets of Fig. 2). We first reconstruct a compressed ghost image of the static frame of the scene, which represents the background. In order to do this, we put 2000 different random patterns on $\mathrm{DMD}_{g}$, with $\mathrm{DMD}_{o}$ displaying the background scene. These realizations 
represent $49 \%$ of a raster scan. For each random pattern, we count coincidence detections for $8 \mathrm{~s}$. Typical single count rates were $13.8 \times 10^{3}$ counts/s for the ghost and object arms with the coincidence counts approximately $2 \%$ of the single counts. Fig. 2(a) shows the retrieved background scene $\hat{O}$. After this, subsequent frames of the scene with the object in different positions are displayed on $\mathrm{DMD}_{o}$. After applying the optimization algorithm, the moving object was clearly identified as shown in Figs. 2(b)-(f). The reconstructions were done using 400 patterns, which represents $9.7 \%$ the measurements of a raster scan. The negative values in the retrieved images are due to background subtraction and fluctuations in the measurements process.

A straightforward examination of the limits of our protocol is carried out by reducing the number of measurements used to track an object. The images shown in Fig. 3 were reconstructed with only 200 and 100 measurements, corresponding to $4.88 \%$ and $2.44 \%$ of the measurements of a raster scan. The metric employed to characterize the fidelity of these reconstructions is the mean-squared error ${ }^{5}$ defined as $M S E=(1 / n)\|O-\hat{O}\|^{2}$. The $M S E$ is seen to increase as the number of measurements is decreased. Although, it is still possible to detect the object trajectory with just 100 measurements.

The photon efficiency is studied by estimating the dependence of the $M S E$ on the num-
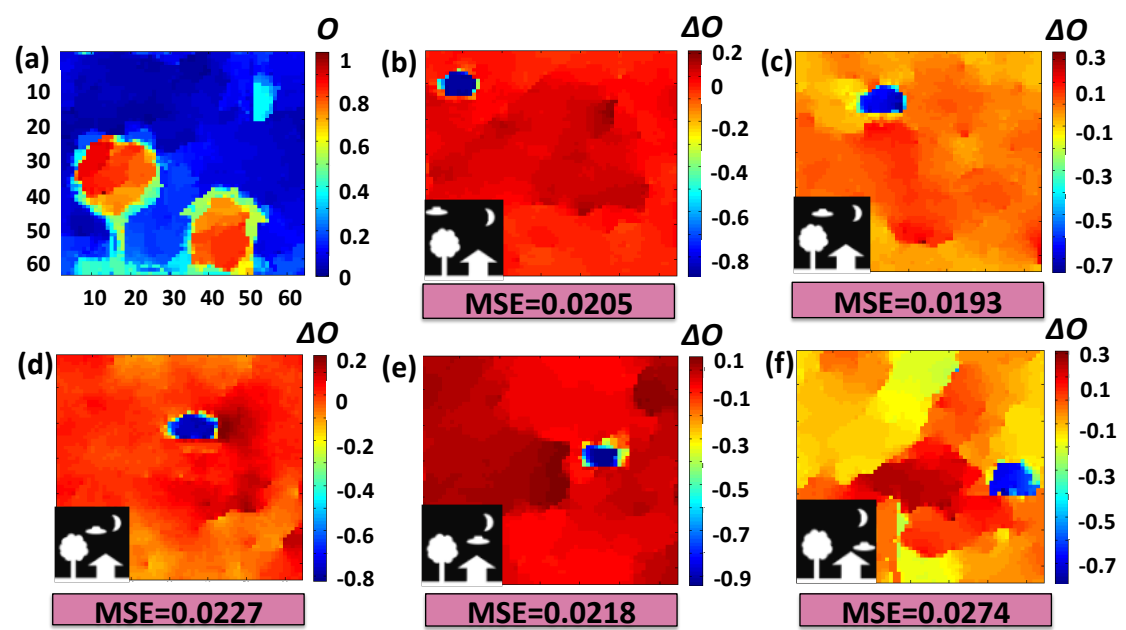

FIG. 2. Compressed ghost image of (a) the background of the scene and (b-f) the tracked object in different positions. These reconstructions were obtained by defining different $\Delta J$ vectors with 400 elements, corresponding to the number of measurements. The insets show the original frames of the scene displayed on the DMD. 
200 measurements

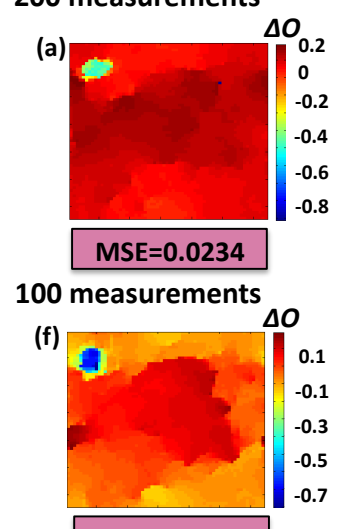

MSE $=0.0293$

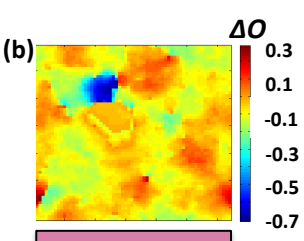

MSE $=0.0249$

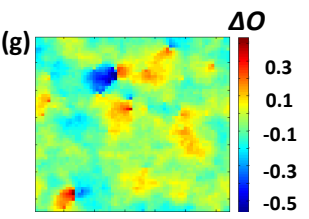

MSE $=0.0295$

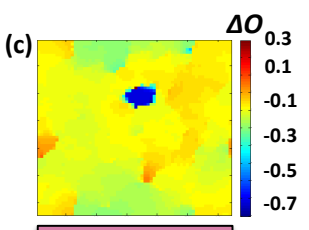

MSE $=0.0257$

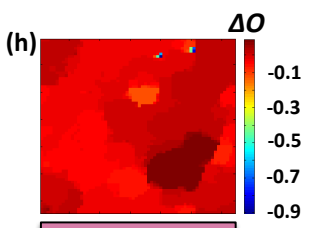

MSE $=0.0335$

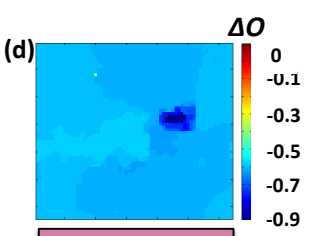

MSE $=0.0225$

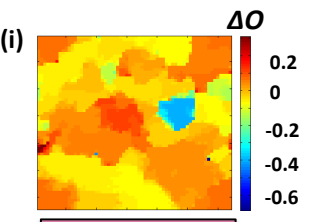

MSE $=0.0291$
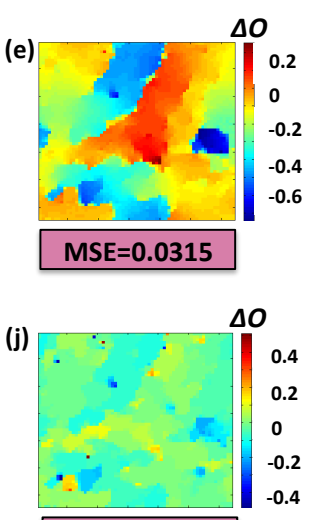

MSE $=0.0395$

FIG. 3. Reconstructed ghost image of (a-e) tracked object with 200 measurements. (f-j) same object with 100 measurements.

ber of photons per measurement, for a fixed number of measurements. A simulation of the protocol was carried out by using the data employed in the experiment. In order to achieve realistic experimental conditions, dark and shot noise were introduced by means of poissonian distributions. The amount of dark noise was modeled based on the frequency distribution of counts obtained when both of the DMDs were turned off. We have considered reconstructions employing 100 and 400 measurements. Fig. 4 shows the dependence of image quality on the number of detected photons per measurement. The minimum number of photons per measurement needed to distinguish the silhouette of the object by eye are 500 photons/measurement and 200 photons/measurement for 100 and 400 measurements respectively. The estimated thresholds correspond to a MSE oscillating around 0.04. For the situation where an object was tracked with 100 measurements and 500 photons/measurement, we estimate that we can impress approximately 0.082 bits/photon. This is considering that for a binary image the number of pixels corresponds to the number of bits ${ }^{25}$.

The maximum object velocity that we can track is limited by the number of photons that we are able to detect. In our setup, each scene reconstruction took 13.3 minutes (for the case of 100 measurements) due to the low photon flux. If we were to use a high brightness source of entangled photons, we could shorten the acquisition time needed to retrieve a compressed ghost image with an MSE below the threshold shown in Fig. 4. As such, there is no hard theoretical limit on the maximum object velocity that can be tracked using this method.

In conclusion, we have proposed and demonstrated a proof-of-principle object-tracking 


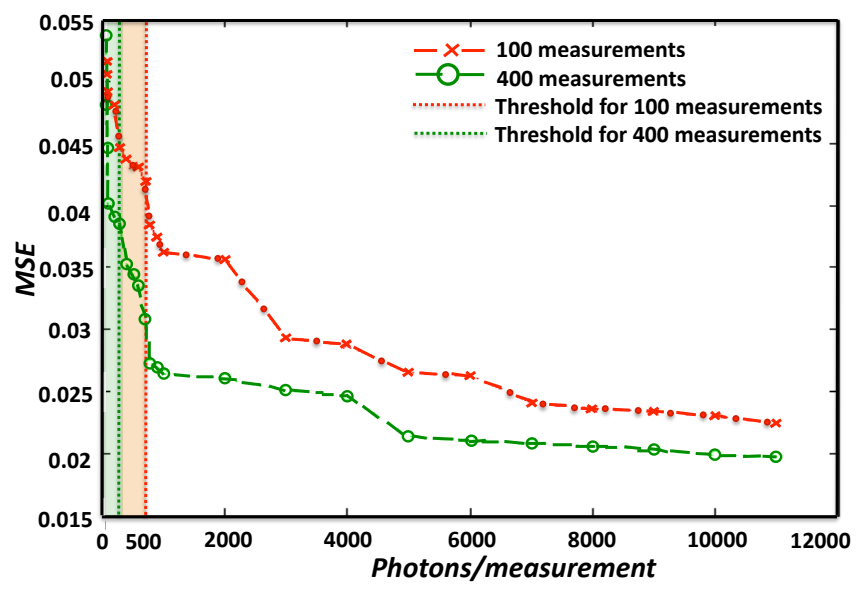

FIG. 4. (Color online) Calculated mean-squared error of the compressed tracked object at the position shown in Fig. 2(b). Green (Red) line indicates the MSE using 400 (100) measurements. The thresholds indicate that a low quality image is retrieved and is not possible to track the object.

protocol in a ghost imaging scheme. This protocol uses compressive sensing to exploit the sparsity existing between two realizations of a scene with a moving object. It also reduces the environmental noise introduced during the measurement process. Further, it allows us to perform image retrieval significantly faster by employing single pixel detectors. Our method is photon-measurement efficient, allowing us to track an object with only $2.44 \%$ of the number of measurements established by the Nyquist criterion, even at low light levels. This economic procedure shows potential for real-life applications.

\section{ACKNOWLEDGMENTS}

The authors would like to thank M. Mirhosseini and A.C. Liapis, P. Ybarra-Reyes and J.J. Sanchez-Mondragon for helpful discussions. This work was supported by the DARPA AFOSR GRANT FA9550-13-1-0019 and the CONACYT.

\section{REFERENCES}

${ }^{1}$ H. Wang, S. Han, and M. I. Kolobov, Optics Express 20, 23235 (2012).

${ }^{2}$ W.-K. Yu, X.-F. Liu, X.-R. Yao, C. Wang, S.-Q. Gao, G.-J. Zhai, Q. Zhao, and M.-L. Ge, arXiv:1202.5866v1 physics.optics (2012). 
${ }^{3}$ G. A. Howland, P. B. Dixon, and J. C. Howell, Appl. Opt. 50, 5917 (2011).

${ }^{4}$ G. A. Howland and J. C. Howell, Physical Review X 3, 011013 (2013).

${ }^{5}$ O. Katz, Y. Bromberg, and Y. Silberberg, Appl. Phys. Lett. 95, 131110 (2009).

${ }^{6}$ P. Zerom, K. W. C. Chan, J. Howell, and R. Boyd, Phys. Rev. A 84, 061804 (2011).

${ }^{7}$ E. J. Candès and T. Tao, Information Theory (2006).

${ }^{8}$ E. J. Candès and M. B. Wakin, Sig. Proc. Mag. (2008).

${ }^{9}$ D. Gross, Y.-K. Liu, S. T. Flammia, S. Becker, and J. Eisert, Phys. Rev. Lett. 105, 150401 (2010).

${ }^{10}$ W. T. Liu, T. Zhang, J. Y. Liu, P. X. Chen, and J. M. Yuan, Phys. Rev. Lett. 108, 170403(2012).

${ }^{11}$ A. K. Jha, M. Malik, and R. W. Boyd, Phys. Rev. Lett. 101, 180405 (2008).

${ }^{12}$ T. Pittman, Y. Shih, D. Strekalov, and A. Sergienko, Phys. Rev. A 52, R3429 (1995).

${ }^{13}$ P. Clemente, V. Durán, V. Torres-Company, E. Tajahuerce, and J. Lancis, Opt. Lett. 35, 2391 (2010).

${ }^{14}$ S. Karmakar and Y. Shih, Phys. Rev. A 81, 033845 (2010).

${ }^{15}$ M. Malik, H. Shin, M. O’Sullivan, P. Zerom, and R. W. Boyd, Phys. Rev. Lett. 104, $163602(2010)$.

${ }^{16}$ W. Gong, C. Zhao, J. Jiao, E. Li, M. Chen, H. Wang, W. Xu, and S. Han, arXiv:1301.5767v1 quant-ph (2013).

${ }^{17}$ J. L. O’Brien, A. Furusawa, and J. Vučković, Nature Photon 3, 687 (2009).

${ }^{18}$ C. E. Shannon, Proc. IRE 37, 10 (1949).

${ }^{19}$ M. Malik, H. Shin, and R. W. Boyd, Laser Science (2011).

${ }^{20}$ R. Bennink, S. Bentley, and R. Boyd, Phys. Rev. Lett. 89, 113601 (2002).

${ }^{21}$ A. Gatti, E. Brambilla, M. Bache, and L. Lugiato, Phys. Rev. Lett. 93, 093602 (2004).

${ }^{22}$ C. Monken, P. Ribeiro, and S. Pádua, Phys. Rev. A 57, 3123 (1998).

${ }^{23}$ R. M. Willett, R. F. Marcia, and J. M. Nichols, Optical Engineering 50, 072601 (2011).

${ }^{24}$ C. Li, Master Thesis, Rice University 1, 92 (2009).

${ }^{25}$ S. Nakadate, T. Yatagai, and H. Saito, Appl. Opt. 19, 1879 (1980). 